\title{
Frost formation with salt
}

\author{
J. Guadarrama-Cetina ${ }^{1}$, A. Mongruel ${ }^{2}$, W. GonzÁlez-Viñas ${ }^{1}$ and D. Beysens ${ }^{2,3,4}$ \\ 1 Department of Physics and Applied Mathematics, University of Navarra - Pamplona, Spain \\ 2 Physique et Mécanique des Milieux Hétérogènes, UMR 7636 CNRS - ESPCI - Université Pierre et \\ Marie Curie - Université Paris Diderot - 10 rue Vauquelin, 75005 Paris, France \\ 3 Service des Basses Températures, CEA-Grenoble \& Université Joseph Fourier - Grenoble, France \\ 4 OPUR - 60 rue Emeriau, 75015 Paris, France
}

received 27 March 2015; accepted in final form 1 June 2015

published online 23 June 2015

PACS 68.03.Fg - Evaporation and condensation of liquids

PACS 68.18. Jk - Phase transitions in liquid thin films

PACS 64.70.-p - Specific phase transitions

\begin{abstract}
The formation of frost in presence of salt $(\mathrm{NaCl})$ crystal is experimentally investigated on a hydrophobic surface. It presents several remarkable features due to the interplay of saltywater saturation pressure evolution, initially lower than the saturation pressure of ice and water, and the percolating propagation of ice dendrites from defects throughout the supercooled water droplet pattern. In particular, it is remarkable that nucleation of supercooled water and/or ice is prevented around the salty drop in a region of inhibited condensation where the substrate remains dry. As condensation proceeds, salt concentration decreases to eventually become lower than ice's, allowing ice dendrites to hit the salty drop. Salty water then melts ice but eventually freezes as an effect of dilution.
\end{abstract}

Introduction. - Ice formation on roads, cars, planes, windmills, electric lines, etc. can cause severe problems to society. Preventing frost and icing is then the object of active applied and fundamental researches. Recent studies have focused on passive antifreeze surfaces modified by nano-patterning (see, e.g., $[1,2]$ and references therein). Active materials, inspired by Nature, which release an anti-icing coating, have also been investigated [3,4]. In contrast with these studies, which deals with sophisticated coatings and substrates, we address in the following the simple situation where the most classical anti-freezing agent, salt, is used on a smooth substrate. Although the anti-icing property of salt has been known for long, to our knowledge the exact process by which a salt crystal acts during frost formation has not been investigated yet. Before melting ice crystals by lowering the freezing temperature, we find below that the first action of salt is to prevent nucleation of water and ice on the substrate.

In order to study the process, we set up a model experiment where we analyze how frost forms on a hydrophobic surface on which a small salt crystal was deposited. The decrease of solidification temperature when adding salt to water depends on a number of factors including the concentration of salt in water and the type of salt in the solution. Sodium chloride $(\mathrm{NaCl})$ can exist in aqueous (water-based) solutions with concentrations ranging from $0 \%$ up to about $23 \%$ by weight $[5-7]$. The maximum concentration, which corresponds to the saturation point for the solution, is only weakly dependent on temperature. The freezing point for a saturated solution is about $-21^{\circ} \mathrm{C}$.

On smooth hydrophobic substrates, frost formation in the absence of salt has been shown to be preceded by the nucleation and growth of supercooled water (SC) droplets [8]. Growth of an isolated droplet by diffusionlimited condensation of water vapor from humid air depends on the local water vapor concentration gradient. In the quasi-static approximation (slow growth) the vapor concentration, obeys the simple law $\Delta p=0$ ( $p$ is the vapor pressure, see, e.g., $[9,10])$. Condensation is considered as the inverse process of evaporation [10]. For a hemispherical droplet of radius $R$, the concentration profile exhibits a hyperbolic variation with respect to the distance $r$ from the drop center:

$$
p(r)=p_{\infty}+\left(p_{s}^{\prime}-p_{\infty}\right)(R / r),
$$

where $p_{\infty}$ is the pressure of water vapor at infinity corresponding to the supersaturation pressure, and $p_{s}^{\prime}$ is the water vapor saturation pressure of the condensed phase (pure water, salty water, ice). There results a $t^{1 / 2}$ evolution 


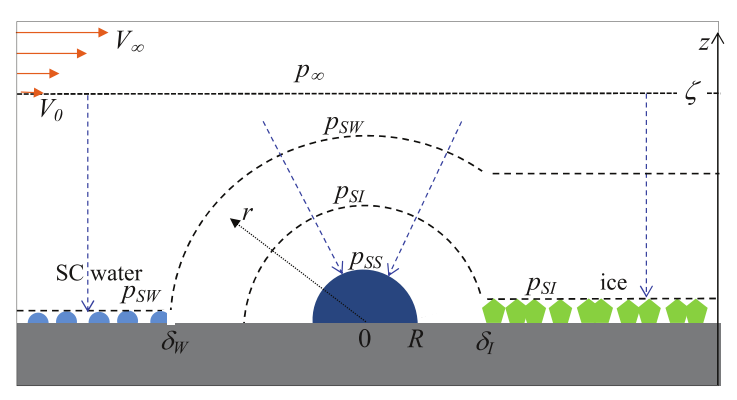

Fig. 1: (Color online) Schematics of condensation of SC water droplets and of frost formation in the vicinity of a salty drop. $\delta_{W}$ and $\delta_{I}$ are the regions of inhibited condensation for SC water and ice, respectively. (For other notations, see text.)

( $t$ is time) of the drop radius. For an assembly of droplets (self-similar growth stage of breath figure BF pattern with constant high surface coverage), the vapor concentration profiles of each drop overlap. The drop pattern can thus be replaced by an equivalent film with mean vapor gradient directed perpendicular to the surface. There results a linear variation for the concentration profile. With $z$ the distance to the substrate

$$
p(z)=p_{s}^{\prime}+\left(p_{\infty}-p_{s}^{\prime}\right)(z / \zeta) .
$$

This equivalent film thickness approximation eventually leads to a $t^{1 / 3}$ evolution for the radius of an individual drop, see, e.g., [9-11]. However, the mean pattern droplet radius $\langle\rho\rangle$ grows as $t$, as an effect of droplets coalescence (see, e.g., [11]). The typical length scale of the vapor gradient, $\zeta$, corresponds to the concentration boundary layer thickness. It is the distance from the substrate where the transport of water molecules by diffusion becomes more efficient than by convection (fig. 1). For a detailed discussion see [11].

Background. - Once the SC droplets have formed a regular breath figure pattern, it has been recently assessed [8] that frost formation proceeds in 2 stages. First, ice nucleates on defects that favor nucleation such as sample edges. The further process depends on the droplet surface coverage. For low surface coverage, crystallization proceeds by the propagation of a front where droplets evaporate on faceted crystals. Ice crystals grow at the expense of liquid droplets because the ice saturation pressure is slightly lower than that of liquid water $[12,13]$. For high surface coverage, droplets still evaporate for the same reason, but the vapor gradient between ice and droplets is large enough to form fast-growing dendrites, which can thus hit a droplet before they completely evaporate. When a droplet is hit, it freezes and emits in turn dendrites, which hit another droplet, and so on, forming a percolating network on the entire surface. The velocity of propagation (or of network formation) depends on surface coverage. The same frost propagation mechanism has been independently discovered by other authors to occur also on superhydrophobic surfaces [14].
On the other hand, the process of dropwise condensation in the vicinity of a salty-water droplet obeys remarkable features [9]. Water condensation from ambient air first dissolves the salt crystal to form a salty drop, initially at salt saturation. The hyperbolic water vapor concentration profile around the salty drop follows eq. (1), with $p_{s}^{\prime}$ replaced by $p_{S S}$, the saturation pressure of salty water. As $p_{S S}$ is always lower than the water vapor pressure, $p_{S W}$, needed for nucleation of water droplets on the substrate (fig. 1), the nucleation of water droplets is impeded inside a ring at distances $r \leq \delta$ from the salty-drop center. This region is called the region of inhibited condensation (RIC) (fig. 1). As time goes on, the radius $R$ of the salty drop increases, and the salt concentration decreases accordingly. It makes $p_{S S}$ to increase and $\delta-R$ to decrease. However, the situation $\delta=R$ can be reached only asymptotically, which is not the case for ice crystals as we develop below. The BF pattern that forms beyond the ring exhibits the typical different stages of growth [9]: nucleation at the RIC perimeter, growth inside the hyperbolic vapor profile (low surface coverage, growth in $t^{1 / 2}$; higher surface coverage, individual droplets growing as $t^{1 / 3}$ corresponding to a linear vapor profile perpendicular to the surface) and self-similar growth with constant high surface coverage and mean droplet radius growing as $t$.

We come now to the case where both processes, SC water droplet condensation and frost formation, occur in the vicinity of a salty droplet. The degree of supersaturation $p_{\infty}-p_{s}^{\prime}$ controls evaporation and condensation. In the present problem, we have to deal with the water vapor pressure of ambient atmosphere, $p_{\infty}(=1950 \mathrm{~Pa}$ at $17^{\circ} \mathrm{C}$ for $100 \%$ relative humidity) and with the $p_{s}^{\prime}$ pressure of water vapor near the condensed phase at the substrate temperature $\left(-12^{\circ} \mathrm{C}\right)$. Owing to local equilibrium assumption, for water, $p_{s}^{\prime}=p_{S W}$; for ice, $p_{s}^{\prime}=p_{S I}$; and for salty water, $p_{s}^{\prime}=p_{S S}$, where the right-hand sides correspond to the equilibrium vapor pressure of the different phases. Note that, in general, nucleation requires supersaturated vapor because of the existence of a nucleation barrier associated to the formation of an interface [15]. However, in the case of complete wetting, the barrier is zero. In our system, at the beginning of the process, supersaturation is large as it corresponds to $p_{\infty}-p_{S W}$ (supercooled water) or $p_{\infty}-p_{S I}$ (ice crystal), which authorizes heterogeneous nucleation on the substrate. Once a drop (or an ice crystal) has nucleated, condensation proceeds through the incorporation of water molecules at the drop or crystal surface. There is no nucleation barrier for this process (it corresponds to complete wetting) and growth proceeds due to the water vapor concentration gradient. There is also no nucleation barrier concerning evaporation from water droplets on ice crystals or salty drop, or evaporation of water on salty drop. Only small supersaturation is needed to ensure a water vapor concentration gradient. Because of continuous water condensation, $p_{S S}$ increases with time from saturated salt pressure $p_{S S 0}$ to water pressure $p_{S W}$, as an effect of 
Table 1: Water saturation pressure under different conditions (from [5-7]).

\begin{tabular}{|c||c|c|c|}
\hline Conditions & $T\left({ }^{\circ} \mathrm{C}\right)$ & Symbol & Value $(\mathrm{Pa})$ \\
\hline \hline $\begin{array}{c}\text { Salty water } \\
\text { (salt saturation) }\end{array}$ & -12 & $p_{S S 0}$ & 180 \\
\hline Salty water & -12 & $p_{S S}$ & $180-245$ \\
\hline Ice & -12 & $p_{S I}$ & 220 \\
\hline Water & -12 & $p_{S W}$ & 245 \\
\hline Humid air & 17 & $p_{\infty}$ & 1950 \\
\hline
\end{tabular}

salt dilution. At $-12{ }^{\circ} \mathrm{C}, p_{S W}=245 \mathrm{~Pa}, p_{S I}=220 \mathrm{~Pa}$, $p_{S S 0}=180 \mathrm{~Pa}[5-7]$ (table 1$)$.

As $p_{\infty}>p_{S W}, p_{S I}, p_{S S 0}, \mathrm{SC}$ water, ice and saturated salt solution will grow at the expense of surrounding humid air. In addition, as $p_{S W}>p_{S I}$, SC water evaporates and re-condenses on ice. On the other hand, both water and ice evaporate and re-condense on salty water as $p_{S I}$, $p_{S W}>p_{S S 0}$. Finally, as long as $p_{S S}<p_{S I}, p_{S W}$, there will be a RIC for ice (of extent $\delta_{I}$ ) and for water (of extent $\left.\delta_{W}\right)$ around the salty drop. Due to condensation-induced salt dilution, after some time the salty drop vapor pressure $p_{S S}$ will become equal to that of ice, $p_{S I}$. It practically means that an ice crystal can hit the salty drop: the RIC of ice vanishes $\left(\delta_{I}=R\right)$.

In the following we address the evolution of the salty drop, the formation of frost and its evolution with the percolating freezing process of SC water droplets.

Experiment details. - We use a plane and smooth hydrophobic substrate obtained by coating a thin glass microscope slide with a hydrophobic film (3M-EGC 1700). The film is obtained manually by the dip-coating method. The contact angle with ultrapure water, as measured by the sessile drop method, is $95^{\circ}$, with advancing angle $108^{\circ}$ and receding receding $82^{\circ}$ at ambient room temperature $\left(21 \pm 0.5^{\circ} \mathrm{C}\right)$. The experimental procedure is the same as already described elsewhere [8]. A clean substrate is fixed on a horizontal electrolytic copper plate in the condensation chamber. The temperature of the copper plate is driven to $-12^{\circ} \mathrm{C}$ using a Peltier device. Air flow saturated with water vapor is obtained by bubbling into ultrapure water cooled with a thermostatic bath set to $+17^{\circ} \mathrm{C}$. This flow is streamed with a constant flux of $2.3 \mathrm{~L} / \mathrm{min}$ through two inlet nozzles placed in front of each other in the chamber, so that a stagnation point is obtained at the center of the chamber, where the slide is set and the observation is performed.

The evolution of the condensed pattern is observed by optical microscopy using a high-resolution CCD color camera PixeLink (PL B776F, $2048 \times 1536$ px.) connected to a computer. The frame rate is $25 \mathrm{fps}$. The objective used was $\times 8$, corresponding to $1.88 \mathrm{px} / \mu \mathrm{m}$. Then the screen width $=2048 / 1.88=1089 \mu \mathrm{m}$. The images are processed and analyzed with MATLAB. a

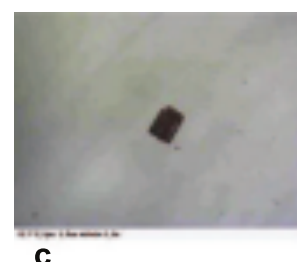

b

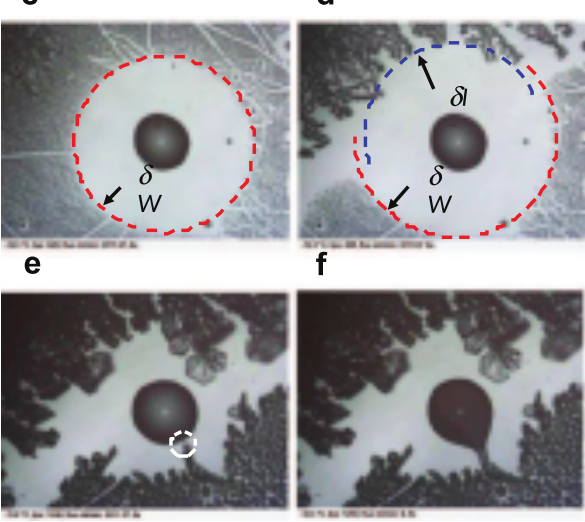

Fig. 2: (Color online) Typical behavior. (a) Salt crystal just after deposition $(t=0)$. (b) Partial crystal dissolution, salty drop at supersaturation $(t=8 \mathrm{~s})$. The small bubble inside is trapped air. (c) Condensation under humid air $(t=30 \mathrm{~s}$; $2.3 \mathrm{~L} / \mathrm{min}$ flow rate; air saturated with water at $17^{\circ} \mathrm{C}$ ) showing the water RIC $\left(\delta_{W}\right)$. Ice is seen to percolate from top left. (d) Icing in the left diagonal $(t=34 \mathrm{~s})$ showing the ice RIC $\left(\delta_{I}\right)$. (e) $40 \mathrm{~ms}$ before an ice dendrite hits the drop (white circle; $t=49.6 \mathrm{~s}$ ). (f) When hit, the salty drop immediately invades ice crystals from the dendrite $(t=50 \mathrm{~s})$.

Observation and discussion. - For each condensation experiment the following procedure has been followed. Initially a $\mathrm{NaCl}$ salt crystal (typical size $100 \mu \mathrm{m}$ ) is deposited on the slide. Then the copper plate is cooled down to $-12{ }^{\circ} \mathrm{C}$. This stage lasts typically $30 \mathrm{~s}$. (fig. 2). When this temperature is reached, water vapor is streamed into the chamber. All condensation experiments follow the same behavior (see the supplementary video silanizedsink0-video.mp4). i) During the cooling process water condensation proceeds on the salt crystal from humid air in the chamber until full crystal dissolution occurs (fig. 2(a), (b)). ii) Then the flow of humid air is sent in the chamber. Condensation of SC water droplets starts around the salty drop outside a region of inhibited condensation (fig. 2(c)) and form a BF pattern. This process is the same as reported in [9]. The water RIC decreases continuously, due to the continuous increase of the water pressure of the salty drop related to its steady growth. iii) During this process, ice nucleation proceeds at one end of the sample (left-hand side top of the sample, fig. 2(c)). Icing propagates rapidly by a percolation process as reported in [8]. In this realization, the left side of the sample freezes first. In fig. 2(d), one can see a region of such icing process, forming an ice RIC, together with a region that has not been frozen yet with its water RIC. The propagation velocity of icing depends on the 


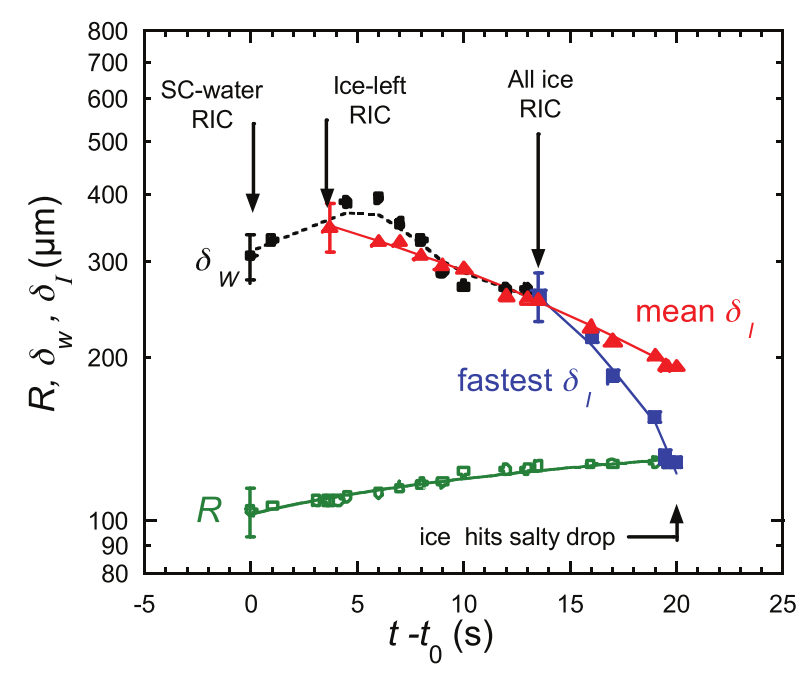

Fig. 3: (Color online) Evolution of RIC radius $\delta_{W}$ (SC water, full circles) and $\delta_{I}$ (ice, triangles) correlated with salty-drop radius $R$ (circles). The lines in $\delta_{W}$ (interrupted) and $\delta_{I}$ (full) are $80 \%$ weighing function. The full line in $R$ is a fit to eq. (4). The squares correspond to the fastest ice propagation that eventually hits the salty drop at radius $R_{c}=128 \mu \mathrm{m}$.

surface coverage. When the icing front is close to the RIC border of the SC water droplets, the droplet surface coverage is lower and those droplets evaporate before being hit by ice. There results a slight growth of the RIC when freezing (fig. 3). Eventually, the other (right side) area of SC water droplets freezes and RIC is only concerned with ice (fig. 2(e)). iv) As the saturation pressure of the salty drop continuously increases with condensation, the ice RIC decreases, till an ice crystal touches the salty drop (fig. 2(e)). This occurs for the salty-drop radius $R_{c}$ and corresponds to a salty-drop pressure equal to the ice pressure. v) Once the crystal has hit the salty drop, the latter wets and melts the crystal (fig. 2(f)) in a time lower than the frame rate $(40 \mathrm{~ms})$. There then results a reduction in salt concentration in the melt that eventually makes it freeze again.

In the following we report and quantify a typical experiment, where SC droplets and ice crystals evolution can be clearly identified.

The evolution of the salty-drop radius corresponds to a supersaturation that decreases as condensation proceeds. With $R_{0}$ the initial radius corresponding to salt saturation, the salty-water saturation pressure approximately follows [9]:

$$
p_{S S}(R) \approx p_{S W}-\left(p_{S W}-p_{S S 0}\right)\left(R_{0} / R\right)^{3},
$$

which leads to the radius evolution

$$
R=\left[R_{0}^{5}+B\left(t-t_{0}\right)\right]^{1 / 5} .
$$

Here $t_{0}(=30 \mathrm{~s})$ corresponds to the time where air flow is sent to the substrate and $B \propto\left(p_{S W}-p_{S S 0}\right) R_{0}^{3}$. Data of fig. 2 experiment are reported in fig. 3. They can be fitted to eq. (4) with $B=(1.3 \pm 0.06) \cdot 10^{9} \mu \mathrm{m}^{5} \mathrm{~s}^{-1}$ and $R_{0}=(103 \pm 1) \mu \mathrm{m}$ (uncertainty: one standard deviation). The latter value compares well with the measured value $R_{0}=104.5 \mu \mathrm{m}$ at $t=t_{0}$.

Ice and water RIC evolution. - The nucleation of $\mathrm{SC}$ water drops at the edge of the RIC results from the interplay at the surface level of the hyperbolic water concentration profile imposed by the salty drop, eq. (1), and the linear profile above the $\mathrm{SC}$ water drop pattern, eq. (2). As can be seen in fig. 2, water droplets or ice crystals are close together, which validates the equivalent film approximation and the linear profile. In ref. [9], the supersaturation was low, the contribution of the linear profile was then small and the salty drop imposed the hyperbolic profile on the surface. In the present situation where the supersaturation is large, both profiles combine in a complex manner. A precise determination of the combined profile is out of the scope of the present work. One can, however, estimate the RIC by a scaling analysis.

The RIC corresponds indeed to the place where vapor condensation from ambient humid air compensates evaporation of water droplets or ice crystals on the salty drop. Let us consider the mass balance in a annular region situated between the distances $r=\delta$ and $r=\delta+\langle\rho\rangle$ from the salty-drop center. Let us consider the drop pattern as equivalent to a film of thickness $h$ [9-11]. This annular region condenses water vapor from ambient at its top horizontal surface $(z=h)$, and evaporates onto the salty drop through the vertical surface situated at $r=\delta$. No mass exchange occurs through the other vertical surface at $r=\delta+\langle\rho\rangle$ as no vapor gradient exists there.

The condensed (or evaporated) mass is proportional to the integral of the vapor gradient on the condensation (or evaporation) surface [8-11]. The vapor gradient for condensation is constant along $z$ and equals $(\nabla p)_{c}=\left(p_{\infty}-p_{S j}\right) / \zeta$. Here $j$ stands for $W$ (water droplets) or $I$ (ice). Condensed mass is then proportional to $\pi\left[(\delta+\langle\rho\rangle)^{2}-\delta^{2}\right](\nabla p)_{c} \approx 2 \pi\langle\rho\rangle \delta(\nabla p)_{c}$. Evaporation proceeds along the vertical internal surface of the annular region and corresponds to the mean vapor gradient $(\nabla p)_{e}=\left(p_{S j}-p_{S S}\right) /(\delta-R)$. It is then proportional to $2 \pi \delta h(\nabla p)_{e}$. By equaling the evaporated- and condensedvapor quantities and noticing that the thickness $h$ of the equivalent film scales as $\langle\rho\rangle$ [11], one gets

$$
\delta-R \approx \zeta \frac{p_{S j}-p_{S S}}{p_{\infty}-p_{S j}} \approx \zeta \frac{p_{S W}-p_{S S 0}}{p_{\infty}-p_{S W}}\left(\frac{R_{0}}{R}\right)^{3} .
$$

Here one has combined eq. (1) with the approximation $p_{S W}-p_{S S 0} \approx p_{S I}-p_{S S 0}$ and $p_{\infty}-p_{S W} \approx p_{\infty}-p_{S I}$. This corresponds to have nearly the same RIC for both $\mathrm{SC}$ water and ice, as is indeed observed. The variation of $\delta^{*}=\delta-R$ with respect to $R / R_{0}$ is shown in fig. 4 . As $\left(p_{S W}-p_{S S 0}\right) /\left(p_{\infty}-p_{S W}\right) \approx 0.038$ it is fitted to $R / R_{0}=$ $0.038 \zeta\left(R_{0} / R\right)^{3}$ leaving $\zeta$ as free parameter. It is found $\zeta=(6.3 \pm 0.4) \mathrm{mm}$ for $\mathrm{SC}$ water and $(6.04 \pm 0.05) \mathrm{mm}$ for ice (uncertainty: one standard deviation). Although this approach is only based on scaling analysis, the value 


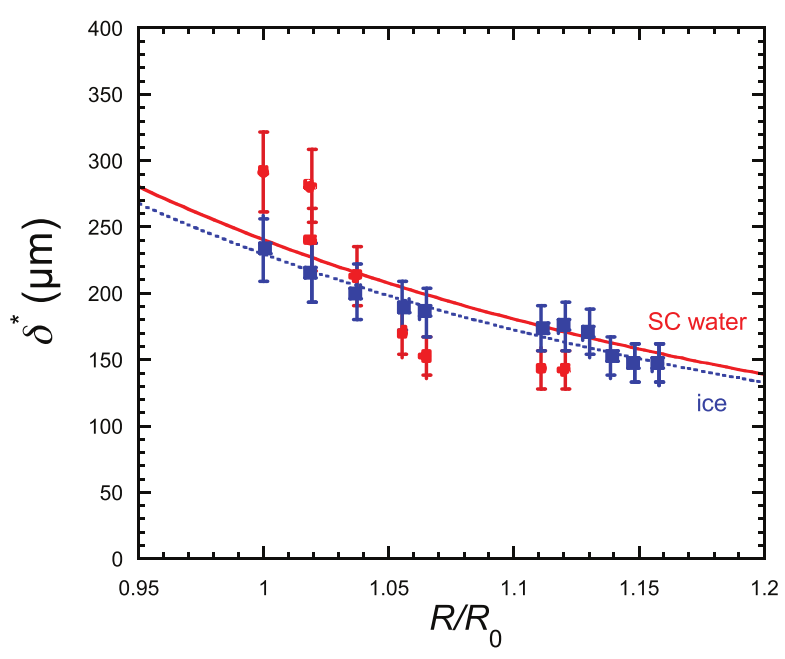

Fig. 4: (Color online) Evolution of the RIC thickness $\delta^{*}=\delta-R$ with respect to $R / R_{0}$. The lines are fits to eq. (5). (SC water: circles, solid line; ice: squares, interrupted line.)

for $\zeta$ on the order of $\mathrm{mm}$, is typical of boundary layer thickness [11].

Hitting the salty drop. - The final situation where an ice dendrite hits the salty drop can be determined by considering the evolution of the salty-drop water pressure, $p_{S S}(R)$ according to eq. (3). The radius $R_{c}$ for which the ice crystal hits the salty drop $\left(\delta=R_{c}\right)$ corresponds to solving eq. (1) with $p_{S S}\left(R_{c}\right)=p_{S I}$. From table 1, one gets the value $R_{c} / R_{0}=1.36 \pm 0.5$, the uncertainty corresponding to a few $\%$ uncertainty on pressure values. This value compares well within uncertainties to the experimental value 1.2 as found in experiment (fig. 3).

Concluding remarks. - The effect of salt on frost formation presents several remarkable features. Initially, salt crystal dissolves by water vapor condensation, forming a water drop at salt saturation. As salty-water saturation pressure is lower than that of water and ice, nucleation of supercooled water and/or ice is prevented around the salty drop in a region of inhibited condensation. Here the substrate remains dry. Outside, supercooled water droplets freeze from a percolating icing process starting from ice crystals that have nucleated on substrate defects. This process develops through the water droplet pattern due to ice saturation pressure lower than water's. As condensation proceeds, salt concentration in the salty drop decreases to become eventually lower than ice's, allowing an ice dendrite to hit it. Then salty water invades the ice pattern, melting it and then eventually freezing as an effect of dilution. It is remarkable that the presence of salt has two effects, lowering the freezing temperature, as it is well known, but also preventing water and ice nucleation, a more subtle effect.

$$
* * *
$$

This work was partly supported by the Spanish Government (MINECO grant No. FIS2014-54101-P). JG-C acknowledges financial support from the "Asociación de Amigos de la Universidad de Navarra".

\section{REFERENCES}

[1] Varanasi K. K., Deng T., Smith J. D., Hsu M. and Bhate N., Appl. Phys. Lett., 97 (2010) 234102.

[2] Xu Q., Li J., Tian J., Zhu J. and Gao X., ACS Appl. Mater. Interfaces, 6 (2014) 8976.

[3] Strom C. S., Liu X. Y. and Jia Z., Biophys. J., 89 (2005) 2618.

[4] Sun X., Damle V. G., Liu S. and Rykaczewski K., Adv. Mater. Interfaces, 2 (2015) 1400479.

[5] Lide D. R. (Editor), CRC Handbook of Chemistry and Physics, 79th edition (CRC Press) 2006.

[6] Sawamura S., Egoshi N., Setoguchi Y. and Matsuo H., Fluid Phase Equilib., 254 (2007) 158.

[7] Arias-González I., Reza J. and Trejo A. J., Chem. Thermodyn., 42 (2010) 1386.

[8] Guadarrama-Cetina J., Mongruel A., GonzÁlez-ViÑas W. and Beysens D., EPL, 101 (2013) 16009.

[9] Guadarrama-Cetina J., Narhe R. D., Beysens D. A. and González-Viñas W., Phys. Rev. E, 89 (2014) 012402 .

[10] Sokuler M., Auernhammer G. K., Liu C. J., Bonaccurso E. and Butt H. J., EPL, 89 (2010) 36004.

[11] Medici M.-G., Mongruel A., Royon L. and Beysens D., Phys. Rev. E, 90 (2014) 062403.

[12] Murphy D. M. and Koop T., Q. J. R. Meteorol. Soc., 131 (2005) 1539.

[13] Cantrell W., Ochshorn E., Kostinski A. and Bozin K., J. Atmos. Ocean. Technol., 25 (2008) 1724.

[14] Boreyko J. B. and Collier C. P., ACS Nano, 7 (2013) 883.

[15] NA B. and WebB L. R., Int. J. Heat Mass Transfer, 46 (2003) 3797. 